\title{
The Use of an Improved Intragastric Balloon Technique to Reduce Weight in Pre-obese Patients-Preliminary Results
}

\author{
Gustavo L. Carvalho • Cesar Borges Barros • Carlos Eduardo Moraes • \\ Masaichi Okazaki • Moacir de Novaes Lima Ferreira • José Sérgio N. Silva • \\ Pedro Paulo C. de Albuquerque • Raphael de Macedo Cavalcanti Coelho
}

Received: 29 April 2009 / Accepted: 10 August 2009 /Published online: 9 September 2009

(C) The Author(s) 2009. This article is published with open access at Springerlink.com

\begin{abstract}
Background In this study, a new safer and faster intragastric balloon procedure was also employed to treat preobese patients (body mass index $(\mathrm{BMI})<30$ ).

Methods From June 2006 to June 2009, 20 such patients were submitted to the Silimed Gastric Balloon treatment, as a part of a multidisciplinary program involving clinical, psychological, and behavioral approaches. For the 16 patients who had completed the 5-6-month treatment, the initial mean weight and mean BMI were $74 \mathrm{~kg}$ and $27.5 \mathrm{~kg} / \mathrm{m}^{2}$, respectively.
\end{abstract}

Gustavo L. Carvalho is a member of SAGES, SOBRACIL, ALACE, ELSA, and Brazilian College of Surgeons (CBC).

\author{
G. L. Carvalho $(\bowtie) \cdot$ M. Okazaki $\cdot$ J. S. N. Silva \\ P. P. C. de Albuquerque $\cdot$ R. de Macedo Cavalcanti Coelho \\ Department of Surgery, Faculdade de Ciências Médicas (FCM), \\ Universidade de Pernambuco (UPE), \\ Av. Boa Viagem 5526B Ap1902, \\ 51030-000 Recife, PE, Brazil \\ e-mail: gc@elogica.com.br \\ e-mail: glcmd1@gmail.com

\section{B. Barros} \\ Clinical Evaluation Department, Silimed, \\ Rio de Janeiro, Brazil \\ C. E. Moraes \\ Clínica Gastrocor, \\ Rio de Janeiro, Brazil \\ M. de Novaes Lima Ferreira \\ UNIPECLIN, Hospital Universitário Oswaldo Cruz (HUOC), \\ Universidade de Pernambuco (UPE), \\ Recife, Brazil
}

Results After the treatment, the mean weight and BMI values decreased significantly to $65.9 \mathrm{~kg}$ and $24.5 \mathrm{~kg} / \mathrm{m}^{2}$, respectively. There were only minor complications - nausea and vomiting - with the exception of two cases of spontaneous deflation of the devices which were successfully removed by gastric endoscopy.

Conclusions Thus, preliminary data suggest that this new intragastric balloon procedure might also be a safe and effective treatment for weight loss in pre-obese patients.

Keywords Gastric balloon Endoscopic procedures . Safety · Efficiency · BMI · EWL · Weight loss · Pre-obese · Overweight

\section{Introduction}

Among the recently improved minimally invasive procedures, silicone intragastric balloon has been a temporary non-surgical option that can promote weight loss in obese patients by partially filling their stomach and inducing a sense of early satiety [1-4].

Recently, a new intragastric balloon technique with technical improvements in the placement and removal procedures, including the use of a new device known as the Silimed Gastric Balloon (SGB), was developed which demonstrated promising preliminary results in a group that was mainly comprised of obese patients [5].

In the present study, the authors present the preliminary use of this new improved procedure in the weight-loss treatment of pre-obese patients (body mass index (BMI) 25.1-29.9) also. The preliminary results obtained are presented and discussed. 


\section{Methods}

Patients

From June 2006 to June 2009, 20 patients with pre-treatment BMI between 25.1 and 29.9 were submitted to treatment with SGB. All of them failed to respond to previous clinical treatment for weight loss. Their mean age was 37 (15-64) years, mean initial weight was $74.6 \pm 9.8(58.3-98.0) \mathrm{kg}$, and mean BMI was $27.6 \pm 2.0(21.6-29.9) \mathrm{kg} / \mathrm{m}^{2}$.

\section{Device}

The SGB design specifications are based on certain requirements defined in the 1987 Tarpon Springs's International Workshop for safety and efficiency of intragastric balloon designs [6].

The SGB is supplied empty, and is delicately rolled up inside a thin silicone sheath. This makes its placement and positioning in the gastric fundus possible by endoscopic route. The device consists of a smooth and transparent silicone shell that acquires a round format when filled with saline solution. The filling is done by a tube with a polytetrafluorethylene needle at its extremity, which is connected to a self-sealing valve attached to the device shell.

\section{Initial Protocol}

The pre-procedure was conducted by a multidisciplinary team. The absolute contraindications were described in an earlier study [5]. Free and informed consents were obtained from each patient.

The SGB placement procedure was immediately preceded by a diagnostic esophagogastroduodenoscopy to define the gastric anatomy, verify any abnormalities contraindicative to SGB placement, and aspirate the gastric content. After removing the endoscope, the SGB was lubricated with surgical lidocaine gel to initiate the insertion procedure.

\section{Improved Placement and Removal Procedures [5]}

Both the procedures were performed under usual sedation of diagnostic endoscopy. During the placement procedure, the extremity of SGB's sheath was carefully anchored to the endoscope extremity using a polypectomy snare (only the extremity of the silicone sheath, and not of the shell, is tied so that the shell of the device does not get damaged). Subsequently, the SGB was smoothly inserted into the stomach by traction under direct visual examination, released by the polypectomy snare near the pylorus and finally positioned in the gastric fundus observed by "J" maneuver, followed by SGB traction by the introduction catheter. In the gastric fundus, the SGB was filled with saline solution (mean of $632 \mathrm{ml}$ ) through direct visual examination and volumes of Iopamiron ${ }^{\circledR}$ contrast $(20 \mathrm{ml})$ and $2 \%$ methylene blue $(10 \mathrm{ml})$ were fixed with the approximate final proportion of $65: 2: 1$. The filling procedure was continually monitored so that a better adequacy of the SGB volume to the gastric capacity was achieved. After the filling procedure, the SGB was visually inspected for the detection of possible deflation or valve malfunctions and confirmation of the correct positioning in the gastric fundus. Antiemetics and antispasmodics were administered orally or intravenously to control nausea, vomiting and pain for 24-72 h. A proton pump inhibitor was prescribed for all the patients during the 6-month treatment. The operational mode of placement procedure is illustrated in an earlier study [5].

The first part of the SGB removal procedure was the positioning of a double silicon overtube previously lubricated with surgical lidocaine gel in the patient's esophagus. Under direct visual observation, a hole was made by a specially developed catheter containing a needle (Scorpion) in each SGB, and the catheter inserted to empty the SGB. Each completely emptied SGB was captured by a polypectomy snare, and also pulled until part of the SGB was held in the overtube. For the very flat balloon a doublehook endoscopic forceps was used to bring the balloon partially inside the esophagus, grasped by the polypectomy snare, allowing the simultaneous removal of the balloon along with the whole endoscopic apparatus (Fig. 1).

\section{Statistics}

To confirm the normal distribution of the efficiency variables, the Shapiro-Wilk's test for normality was used. The test $t$ for paired observations under the significance of

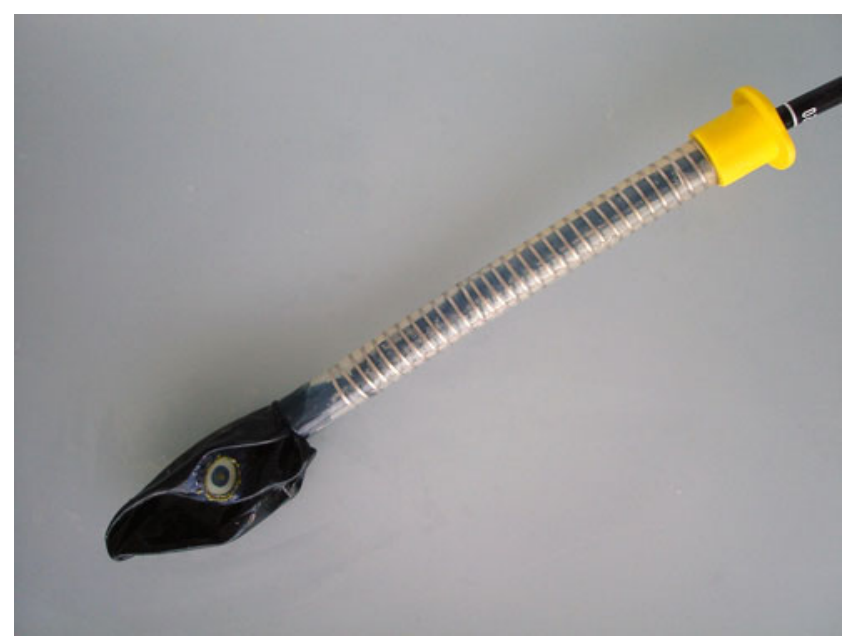

Fig. 1 Final stage of the SGB removal procedure, when the completely deflated balloon is removed along with the whole endoscopic apparatus 
0.01 was used to evaluate the preliminary effectiveness of the proposed treatment. The descriptive statistics values were presented as mean \pm standard deviation.

\section{Results}

In all the cases, SGBs were successfully placed and removed under usual sedation of diagnostic endoscopy. All but two of the 16 patients who had completed the 5-6-month treatment demonstrated good weight loss at the end of the treatment. The mean final weight was $65.9 \pm 9.4(49.3-81.9) \mathrm{kg}$ and the mean final BMI was $24.5 \pm 2.6$ (21.6-28.5) kg/m² (Fig. 2). These preliminary results were statistically significant.

The only initial complications were episodes of nausea, vomiting, and epigastric pain. Epigastric pain and severe vomiting occurred in two patients, leading to early termination of the treatment. There was no occurrence of late complications, such as severe esophagitis, peptic ulcer,

a

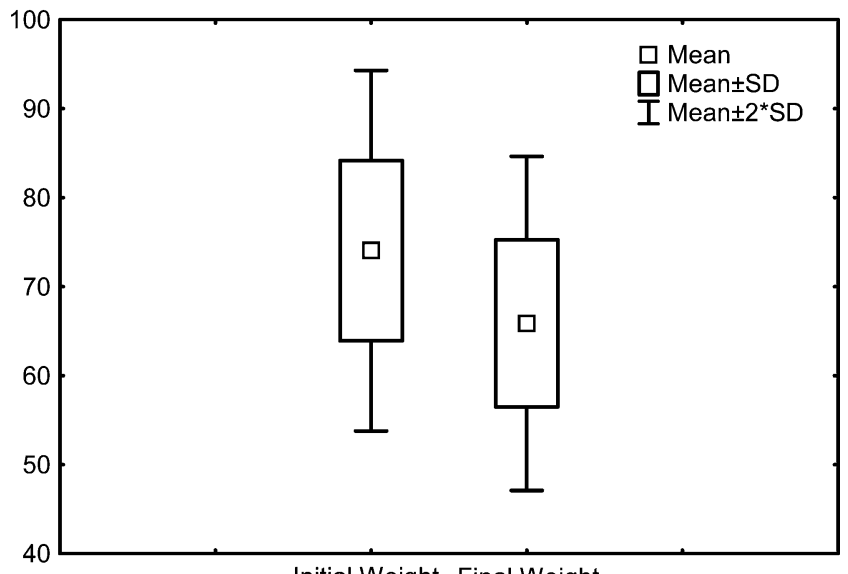

b

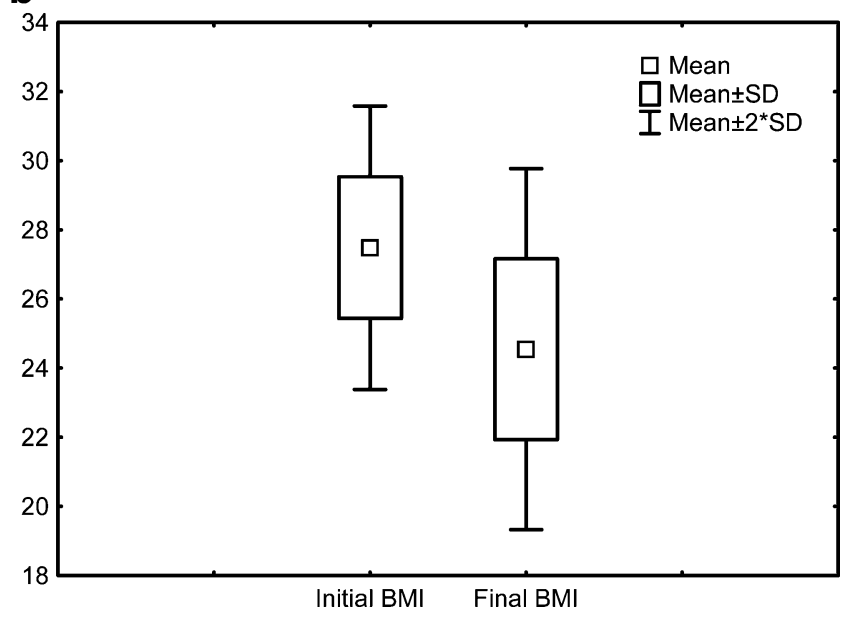

Fig. 2 a, b Boxplots showing the means and dispersions of the initial and final data of the 12 patients who had completed the 6-month treatment with SGB and gastric perforation or erosion. The only major complications were two cases of spontaneous deflation of the devices which were removed uneventfully by office endoscopy.

\section{Discussion}

The use of an intragastric device to control weight in obese patients was first described in 1982 [7]. However, the first gastric balloons did not yield the expected results of weight loss, mainly owing to the technical aspects of the devices' design such as the filling of the devices with air and the presence of a low-resistant balloon shell with rough surface.

In 1999, a new silicone gastric balloon filled with saline solution was presented and recommended for 6-months usage [8], and ever since, significant results with this device have been obtained [1-4]. Recently, a series of technical improvements in the intragastric balloon procedure using a new balloon-SGB - was proposed, and encouraging preliminary results of safety and efficiency in obese patients were obtained [5]. Based on this initial success, the objective of the present study was to use the new SGB technique in the weight-loss treatment of pre-obese patients.

In this series, the improved procedures of placement and removal of the new saline solution-filled balloon also proved to be safer and faster: all the SGBs were successfully placed and removed by office endoscopy, with no intercurrences during the procedures. Also, these procedures were perfectly feasible under the usual sedation of diagnostic endoscopy in ambulatory level, thus avoiding the risks and costs of general anesthesia and operation room. The other main innovations of this new technique include the simplicity and shortened duration of the placement and removal procedures, decrease of pharynx irritation during the placement procedure, better radiopaque visualization of the device along the treatment, and minimal risk of damage to the esophagus or tracheal aspiration or device loss in the digestive tract during the removal procedure [5].

The careful anchoring of the extremity of the SGB's sheath to the extremity of the endoscope made the placement of the device by traction possible, and appeared to be simpler and more effective than the placement by a tube that pushes the device without visual monitoring, as occurs in the usual placement procedure of modern intragastric balloons. The direct visualization enabled the fast positioning of the SGB in the gastric fundus, thereby reducing the excessive manipulation of the endoscope and the consequent risk of damage to the pharynx.

Although SGB has a radiopaque mark around the valve, the use of Iopamiron ${ }^{\circledR}$ in the filling solution of the device contributes to obtaining more clearly defined images on the correct placement of the balloon, whenever necessary. 
The stable catch of the SGB by the polipectomy snare, followed by the joining of SGB directly to the overtube, and the simultaneous removal of the whole endoscopic apparatus, constituted a very safe and effective removal procedure in all cases, as occurred in the initial series [5]. Even in the high pressure area of the esophagus, at the level of the cricopharyngeal muscle, the overtube containing the SGB passed through easily, thus minimizing the risk of damage to the esophagus or device loss in the digestive tract. The use of the overtube practically annulled the risk of tracheal aspiration of saline solution or food residues, thus making the procedure of tracheal intubation unnecessary for controlling these risks. Furthermore, the shortened removal time of SGB resulted in using fewer antispasmodics and decreasing thereby the patient's discomfort, mainly due to lower transparietal stimulation by the endoscopic apparatus and consequent reduction of the spams of the cardia and the esophagus.

In this series, there was no occurrence of any serious complications, such as erosion or peptic ulcer, except for two cases of spontaneous deflation of the balloon (one occurred after 6 months of treatment). In both cases, the deflated devices were successfully removed by gastric endoscopy.

All but two of the 16 pre-obese patients who completed the treatment with SGB lost weight significantly, the mean loss being $8.1 \pm 4.7 \mathrm{~kg}$. The use of $t$ test for paired observations under significance of 0.01 showed that these preliminary results of weight loss in this group of patients were significant. However, the possibility of placebo effect or weight loss due only to behavioral changes cannot be excluded. Nonetheless, two aspects that reinforce the effectiveness of SGB merit emphasis: (1) the recent publication of Genco et al. [4] showing that the modern concept of saline solution-filled intragastric balloon in the weight-loss treatment of obese patients is more effective than the sham procedure associated with restricted diet and (2) the failure history of the patients of the present series to previous clinical treatments for weight loss.

Once the preliminary mean weight loss achieved in the present series is more expressive than those presented by randomized controlled studies using orlistat, sibutramine, and rimonabant to reduce weight in pre-obese patients [9, 10], we think that the SGB technique may act as an alternative to weight-loss treatment with drugs for these patients. Further analysis of a more representative sample will be necessary to test this hypothesis.

Thereby, these early results reinforce the concept of efficacy and safety of the SGB treatment, and suggest a favorable balance between benefits and risks even in preobese patients.

Open Access This article is distributed under the terms of the Creative Commons Attribution Noncommercial License which permits any noncommercial use, distribution, and reproduction in any medium, provided the original author(s) and source are credited.

\section{References}

1. Evans JD, Scott MH. Intragastric balloon in the treatment of patients with morbid obesity. Br J Surg. 2001;88:1245-8.

2. Genco A, Bruni T, Doldi SB, et al. Bioenterics intragastric balloon: the Italian experience with 2515 patients. Obes Surg. 2005; 15:1161-4.

3. Genco A, Cipriano M, Bacci V, et al. Bioenterics Intragastric Balloon (BIB ${ }^{\mathbb{R}}$ ): a short-term, double-blind, randomized, controlled, crossover study on weight reduction in morbidly obese patients. Int J Obes. 2006;30:129-33.

4. Sallet JA, Marchesini JB, Dyker SP, et al. Brazilian multicenter of the intragastric balloon. Obes Surg. 2004;14:991-8.

5. Carvalho GL, Barros CB, Okazaki M, et al. An improved balloon procedure using a new balloon: preliminary analysis of safety and efficiency. Obes Surg. 2009;19:237-42.

6. Schapiro M, Benjamin S, Blackburn G, et al. Obesity and the gastric balloon: a comprehensive workshop. Gastroint Endosc. 1987;33:323-7.

7. Nieben OG, Harboe H. Intragastric balloon as an artificial bezoar for treatment of obesity. Lancet. 1982;1:198-9.

8. Bioenterics Intragastric Balloon (BIB $\left.{ }^{\circledR}\right)$ System. Revisions to Indications for Use. Manufacturer's guidelines information sheet. Carpinteria, California: Bioenterics Corporation, 1999.

9. Padwal RS, Majumdar SR. Drug treatments for obesity: orlistat, sibutramine, and rimonabant. Lancet. 2007;369:71-7.

10. Rucker D, Padwal R, Li SK, et al. Long term pharmacotheraphy for obesity and overweight: updated meta-analysis. BMJ. 2007;335:1194-9. 